\title{
Photoluminescence of short-period GaAs/AlAs superlattices: A hydrostatic pressure and temperature study
}

\author{
S. Guha, Q. Cai, M. Chandrasekhar, and H. R. Chandrasekhar \\ Department of Physics and Astronomy, University of Missouri-Columbia, Columbia, Missouri 65211 \\ Hyunjung Kim, A. D. Alvarenga, R. Vogelgesang, and A. K. Ramdas \\ Department of Physics, Purdue University, West Lafayette, Indiana 47907 \\ M. R. Melloch \\ School of Electrical and Computer Engineering, Purdue University, West Lafayette, Indiana 47907
}

(Received 26 February 1998)

\begin{abstract}
The temperature and pressure dependence of type-I and -II transitions from photoluminescence (PL) spectra in a series of $(\mathrm{GaAs})_{m} /(\mathrm{AlAs})_{m}$ superlattices show that the temperature dependence of energy bands can be described very well with a Bose-Einstein-type equation. From these measurements the parameters that describe the temperature dependence of excitonic transition energies and the corresponding broadening of the PL line are deduced. The pressure dependence of the PL linewidths of the type-I exciton as a function of pressure and temperature yield the intervalley deformation potential. Beyond the type-I-type-II crossover, the PL linewidth increases as a function of both pressure and temperature. The electron-phonon deformation potential for $\Gamma-X$ scattering is found to be temperature dependent. [S0163-1829(98)01935-3]
\end{abstract}

\section{INTRODUCTION}

Carrier dynamics and recombination mechanisms in semiconductor superlattices, in particular those with a staggered band alignment, have been the subject of many investigations. ${ }^{1}$ High-quality superlattices comprising alternating layers of GaAs and AlAs fabricated by epitaxial techniques with monolayer precision are of much interest, thanks to the possibility of designing many physical situations that develop as a result of dimensional changes. Combined with external perturbations such as temperature and pressure, one can tune the electronic states with respect to one another, thus revealing the nature and extent of various interactions. ${ }^{2}$

In Fig. 1, we display the nature of the band alignments associated with the $\Gamma$ valence-band maxima and conductionband minima at $\Gamma$ as well as $X$. When alternating slabs of GaAs and AlAs are grown epitaxially on a [typically (001)oriented] GaAs substrate, the band alignment at the interfaces leads to potential wells for the electrons and holes. As shown in Fig. 1, the conduction band minima associated with the $\Gamma$ point in GaAs and AlAs form potential wells for electrons in GaAs slabs. Similarly, the potential wells in the valence band for holes (only heavy-hole bands are shown) reside in GaAs slabs. The ratio of the depths of these potential wells, known as the band-offset ratio $\Delta E_{c}: \Delta E_{v}$, is an important quantity which has been investigated very extensively via theory and experiment. The potential wells for electrons formed at the $X$-point conduction minima, however, reside in AlAs slabs. This staggered or type-II alignment has the feature that recombination of charge carriers across the heterointerface provides another channel, often in competition with the usual type-I transition across the valence and conduction subbands in GaAs. The energies of the type-I and -II transitions depend on the material parameters of bulk GaAs and AlAs, and the confinement energies of electron and hole superlattice subbands. The latter are governed by the carrier masses, band offset, and the slab thickness of GaAs and AlAs. A calculation ${ }^{3}$ taking into account the accepted values of these parameters ${ }^{4}$ is shown in Fig. 2, where the difference in energy between type-I and -II transitions, $\Delta E$, is plotted for various slab thicknesses (in units of monolayers) of GaAs and AlAs. In the figure, each of the ten curves is for a given $(\mathrm{GaAs})_{m}$ as a function of (AlAs) ${ }_{n}$ where $m$ and $n$ are the number of monolayers of GaAs and AlAs, respectively. Note the curves cross the $\Delta E=0$ line, above which type-II electronic transitions dominate, whereas type-I transitions are dominant below the $\Delta E=0$ line. The type-I to type-II crossover occurs for asymmetric combinations of $m$ and $n$, for example $(2,1),(4,2),(6,3),(9,5)$, and $(10,7)$. For symmetric combinations, the crossover occurs for $m=n=12$.

Studies under hydrostatic stress have proven to be invaluable in the context of the optical properties of semiconductors and their heterostructures. ${ }^{5-8}$ For a given superlattice structure, the difference in energy between the type-I and -II transitions, $\Delta E$, can be tuned with external hydrostatic pressure in a continuous and reversible manner, thus enabling an elucidation of the properties of various interband transitions. ${ }^{9,10}$ Another motivation for pressure studies is to gain insight into intervalley scattering rates ${ }^{11,12}$ which play an important role in relaxation of photoexcited carriers and high-field transport.

The electronic states of semiconductors and heterostructures display large shifts and lifetime-induced broadenings as a function of temperature. ${ }^{2,13}$ In this paper we have investigated photoluminescence (PL) as a function of temperature and pressure of $(\mathrm{GaAs})_{m} /(\mathrm{AlAs})_{n}$ superlattices with $n=m$ $=4,5,10$, and 20. Section II describes our experimental setup. In Sec. III, we show that the temperature dependence 


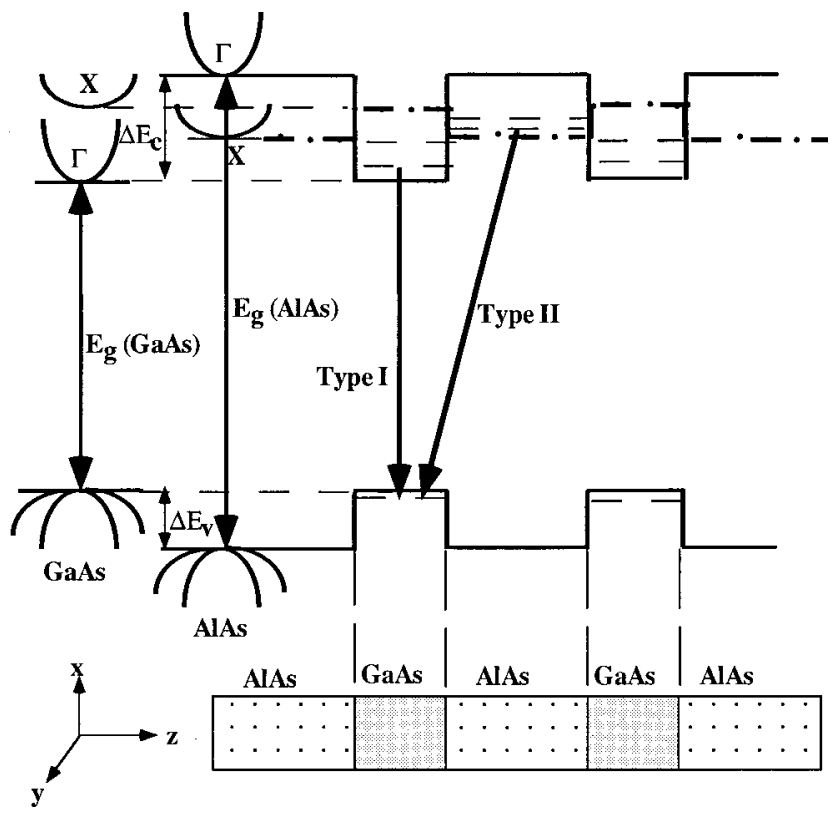

FIG. 1. Schematic band alignment of the $\Gamma, X$, and heavy-hole valence-band extrema of a $(\mathrm{GaAs})_{m} /(\mathrm{AlAs})_{n}$, superlattice. The $\Gamma$ and $X$ bands cross at a pressure $P_{C}$ which depends on the well width. For $P>P_{C}$, the energy of the electrons in the $X$ well of AlAs is lower than that in the $\Gamma$ well of GaAs, and the type-II transition becomes observable.

of the interband excitonic transition energies can be fitted very well with a Bose-Einstein-type equation which was proposed in Ref. 14 to explain the temperature dependence of energy bands in Ge. In Sec. IV, we focus on the pressure dependence of type-I and -II transitions. In Sec. V, the results on the PL linewidths as a function of pressure are presented. We calculate the intervalley electron-phonon deformation potential from our experimental results, and show that the deformation potential changes with temperature.

\section{EXPERIMENT}

$(\mathrm{GaAs})_{m} /(\mathrm{AlAs})_{m}$ superlattices with $m=4,5,10$, and 20 were grown on a (001) GaAs substrate by molecular-beam epitaxy, growth temperatures being $600{ }^{\circ} \mathrm{C}$. The $(10,10)$ and $(20,20)$ superlattices were studied under hydrostatic pressure employing a diamond-anvil cell (DAC); for these measurements, the substrates were thinned to total thickness of $\sim 50$ $\mu \mathrm{m}$. Argon was used as a pressure transmitting fluid in the DAC. Pressure $(P)$ was measured using the luminescence of a ruby chip located in the pressure chamber. The PL signature from the GaAs substrate also serves as a good calibration for pressure since the direct band gap of GaAs moves at the rate of $10.7 \mathrm{meV} / \mathrm{kbar} .{ }^{11}$ The PL spectra were excited using the 514.5-nm $(m=5,10,20)$ and 457.9-nm $(m=4)$ lines of an $\mathrm{Ar}^{+}$laser. The emitted radiation was analyzed with a SPEX 0.85-m double monochromator equipped with a cooled GaAs photomultiplier tube and standard photoncounting electronics. For the low temperature work with DAC a closed-cycle helium refrigerator was employed. The temperature dependence of PL at ambient pressure and in the temperature range 5-300 $\mathrm{K}$ was investigated with a variable temperature cryostat.

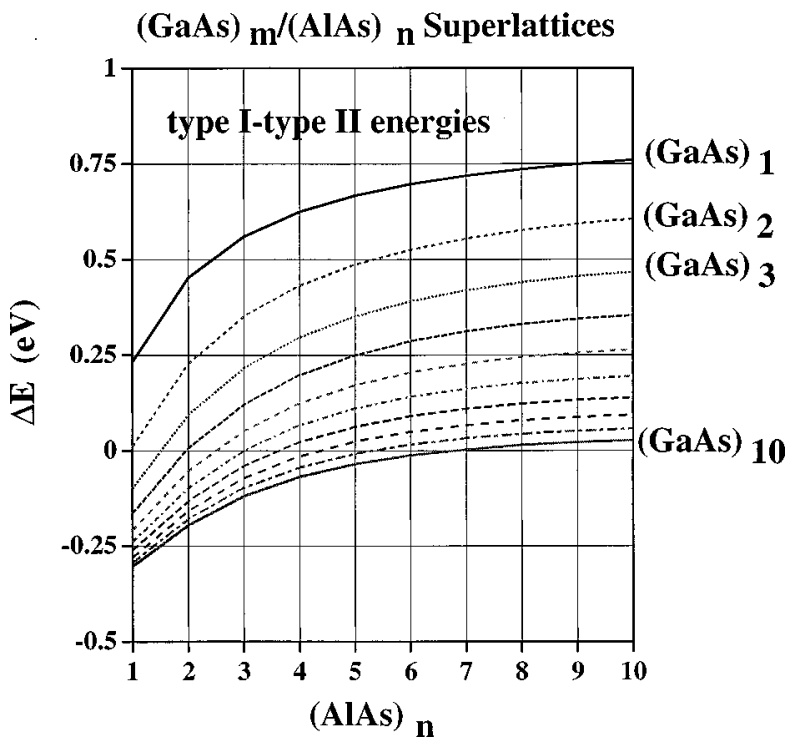

FIG. 2. Difference in energy between type-I and -II transitions, $\Delta E$, for various slab thickness (in units of monolayers) of GaAs and AlAs. Above the $\Delta E=0$ line, the electronic transitions are dominated by lower-lying type-II transitions, whereas type-I transitions are dominant below the $\Delta E=0$ line.

\section{TEMPERATURE DEPENDENCE OF TRANSITION ENERGIES}

As in bulk semiconductors, the electronic energies in heterostructures display a temperature dependence at constant pressure subject to two mechanisms: (1) thermal expansion and (2) renormalization of band energies by electron-phonon interactions. The effect of thermal expansion accounts for a fraction $\left(\sim \frac{1}{4}\right)$ of the total shift, the remaining temperature dependence of band-gap energies arises from electronphonon interactions. ${ }^{15}$ The electron-phonon interactions in turn are of two types: the first-order interaction considered in second-order perturbation (self-energy terms) (Ref. 16) and quadratic interaction taken in the first order (Debye-Waller term). ${ }^{17}$ The temperature-induced change in the band-gap energy $E_{g}$ is usually described by Varshni's empirical expression $^{18}$

$$
E_{g}(T)=E_{g}(0)-\frac{\alpha T^{2}}{T+\beta},
$$

where $E_{g}(0)$ is the band-gap energy at $0 \mathrm{~K}, \alpha$ and $\beta$ are constants for a given semiconductor, the latter being proportional to the Debye temperature. The temperature dependence of the interband transition can be alternately described with an expression in which the energy thresholds decrease proportional to the Bose-Einstein statistical factors for phonon emission plus absorption: ${ }^{14}$

$$
E_{g}(T)=a-b\left(1+\frac{2}{e^{\Theta_{B} / T}-1}\right) .
$$

Here $(a-b)$ is the band gap energy at $0 \mathrm{~K}$, and $b$ represents the strength of the exciton-average phonon interaction. It has 


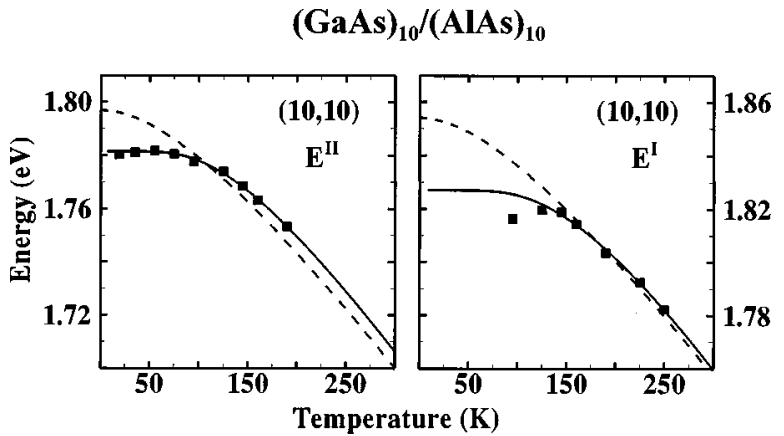

FIG. 3. Temperature dependence of $E^{\mathrm{II}}$ and $E^{\mathrm{I}}$ in $(\mathrm{GaAs})_{10} /(\mathrm{AlAs})_{10}$ superlattices. Experimental data points are shown with solid squares. The dashed curves are fits to Varshni's empirical model [Eq. (1)], whereas the full curves are fits to a Bose-Einstein-type equation [Eq. (2)].

been shown from calculations of electron-phonon spectral functions in $\mathrm{Si}$ and Ge that energy shifts due to the electronphonon interaction include contributions from both acoustic and optical phonons. ${ }^{19}$ Thus $\Theta_{B}$ in Eq. (2) should be viewed as the average frequency of both acoustic and optical branches. The temperature dependence of the linewidth of the interband transitions of semiconductors can be expressed as

$$
\Gamma(T)=\Gamma(0)+\frac{\Gamma_{\mathrm{ep}}}{\left[\exp \left(\Theta_{\mathrm{LO}} / T\right)-1\right]} .
$$

$\Gamma(0)$ represents the broadening due to temperatureindependent mechanisms (such as impurity, dislocation, and surface scattering) and $\Gamma_{\mathrm{ep}}$, the strength of the exciton-LOphonon coupling. Here $\Theta_{\mathrm{LO}}$ is the average frequency of the longitudinal optical phonons. From Ref. 19 it is noted that the optical phonons are the main contributors to the broadening of critical points. This leads to a higher average phonon frequency $\Theta_{\mathrm{LO}}$ from the fit of the broadening parameters to Eq. (3) than for the electron-phonon contribution of the energy shifts with temperature.

Figures 3 and 4 show the energy of the type-I and -II transitions as a function of temperature for $(\mathrm{GaAs})_{m} /(\mathrm{AlAs})_{m}$, where $m=4,5,10$, and 20 ; the squares are the experimental results. The dashed line is a fit to Varshni's empirical model, the values of $\alpha$ and $\beta$ used here being the same as for bulk GaAs, viz. $5.4 \times 10^{-4} \mathrm{eV} / \mathrm{K}$ and $204 \mathrm{~K}$, respectively, while the solid line is a fit to Eq. (2). The following should be noted: $m=4$ and 5 superlattices are type II;

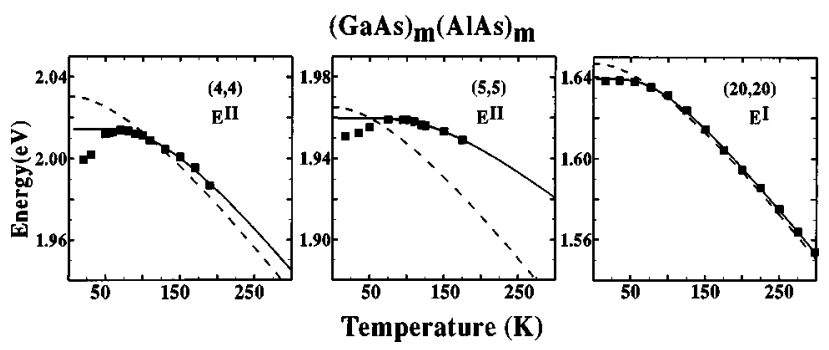

FIG. 4. Temperature dependence of $E^{\mathrm{II}}$ and $E^{\mathrm{I}}$ in $(\mathrm{GaAs})_{m} /(\mathrm{AlAs})_{m}$ superlattices (solid squares). The dashed curves are fits to Varshni's empirical model, and the full curves are fits to a Bose-Einstein-type equation [Eq. (2)], respectively.

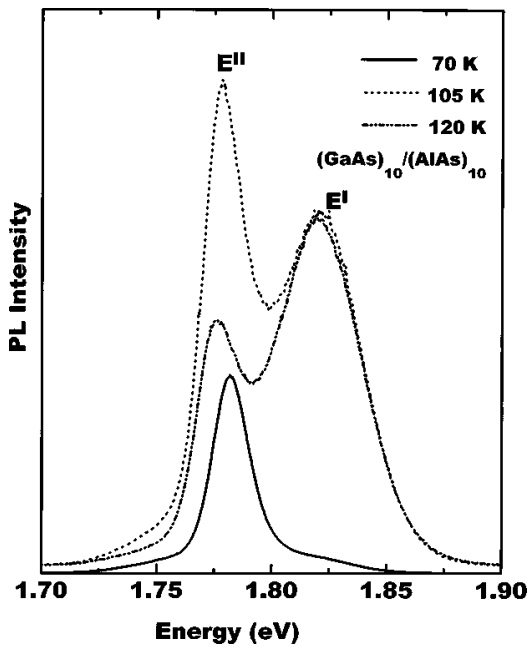

FIG. 5. PL spectrum of a $(10,10)$ superlattice at three different temperatures ( 1 bar). At temperatures above $100 \mathrm{~K}$, the quantumconfined $\Gamma$ level is increasingly populated and the type-I transition is dominant.

the $m=20$ superlattice is type I, and the corresponding transition is dominant up to $300 \mathrm{~K}$. For $m=10$, the type-I and -II transitions lie very close. For this sample, the type-II transition is dominant in the low-temperature PL; at higher temperatures, the quantum-confined $\Gamma$ level is increasingly populated, resulting in the corresponding increase in the intensity of the type-I transition. The PL spectrum of the $(10,10)$ superlattice at 70,105 , and $120 \mathrm{~K}$ shows the appearance of a high-energy peak $E^{\mathrm{I}}$ at the highest temperature (Fig. 5). With increasing temperature the intensity of the low-energy peak $\left(E^{\mathrm{II}}\right)$ decreases, and finally only $E^{\mathrm{I}}$ is observed above $180 \mathrm{~K}$.

In the $(4,4)$ and $(5,5)$ superlattices, an initial increase in energy of $E^{\mathrm{II}}$ is observed up to $80 \mathrm{~K}$. Nakayama et al. ${ }^{20}$ reported a similar initial increase in the energy $E^{\mathrm{II}}$ with a rise in temperature for short-period GaAs/AlAs superlattices. This behavior can be ascribed to the trapping of excitons in localized states resulting from the local fluctuation of the well width along the interface between the wells and the barriers. In this case PL monitors the recombination of excitons occupying a range of energies reflecting the temperature; at low temperatures the lower energies are favored, thus exhibiting an anomalous temperature dependence of the PL energy. Such an anomaly should be particularly visible in a narrow-well superlattice with its larger number of interfaces. At higher temperatures the PL energy shows the usual redshift with increasing temperatures. Anomalies in the temperature dependence of the PL energy have been observed in $\mathrm{Ga}_{x} \mathrm{In}_{1-x} \mathrm{P}$ (Refs. 21 and 22) and $\mathrm{Al}_{x} \mathrm{In}_{1-x} \mathrm{P} \mathrm{Ga}_{x} \mathrm{In}_{1-x} \mathrm{P} /$ $\mathrm{Al}_{x} \mathrm{In}_{1-x} \mathrm{P}$ quantum wells ${ }^{23}$ at low temperatures.

The experimental results in the present investigation are represented better by Eq. (2) rather than by Eq. (1); for the $(5,5)$ superlattice there is almost a $30-\mathrm{meV}$ difference between experiment and the fit from Varshni's model. The $(5,5)$ sample shows the largest deviation from Varshni's empirical model. This may be due to monolayer fluctuations. Fujimoto et al. showed from energy-band calculations that a monolayer fluctuation of GaAs can result in about 50-80meV shifts in the energy gaps. ${ }^{24}$ The $(5,5)$ sample also shows 
TABLE I. Values of the parameters obtained by fitting the interband transition energy vs temperature to Eq. (2).

\begin{tabular}{lccr}
\hline \hline Sample & $a(\mathrm{eV})$ & $b(\mathrm{eV})$ & $\theta_{B}(\mathrm{~K})$ \\
\hline$(4,4) E^{\mathrm{II}}$ & $2.116 \pm 0.025$ & $0.101 \pm 0.026$ & $408 \pm 44$ \\
$(5,5) E^{\mathrm{II}}$ & $2.038 \pm 0.030$ & $0.078 \pm 0.031$ & $484 \pm 67$ \\
$(10,10) E^{\mathrm{II}}$ & $1.897 \pm 0.025$ & $0.115 \pm 0.025$ & $422 \pm 37$ \\
$(10,10) E^{\mathrm{I}}$ & $1.703 \pm 0.064$ & $0.152 \pm 0.031$ & $512 \pm 29$ \\
$(20,20) E^{\mathrm{I}}$ & $1.703 \pm 0.064$ & $0.063 \pm 0.004$ & $272 \pm 12$ \\
Bulk GaAs $E^{\mathrm{Ia}}$ & 1.571 & 0.057 & 240 \\
\hline \hline
\end{tabular}

${ }^{\mathrm{a}}$ From Ref. 26.

the largest broadening of the PL peak, which implies that the energy levels are broadened compared to the other samples. The PL energy as a function of temperature shows an average energy of the broadened band due to which this sample shows a large deviation from Varshni's model. The parameters deduced from fits to Eqs. (2) and (3) are listed in Tables I and II. The temperature dependence of type-I transitions in $(\mathrm{GaAs})_{15} /(\mathrm{AlAs})_{9}$ was studied in Ref. 25, where the authors found a good agreement with Varshni's empirical model. This is expected for the type-I transitions, as can be seen from our result for $(10,10)$ and $(20,20)$; the fits based on both equations coincide above $50 \mathrm{~K}$ for $(20,20)$ and $150 \mathrm{~K}$ for $(10,10)$. Similarly, it is not surprising that the temperature dependence of the PL in a type-I superlattice consisting of GaAs- $\mathrm{Ga}_{1-x} \mathrm{Al}_{x} \mathrm{As}$ superlattice ${ }^{13}$ fits Varshni's empirical model.

The $b$ and $\Theta_{B}$ value (Table I) for the direct transition of the $(20,20)$ superlattice are similar to those of the direct transition in bulk GaAs. ${ }^{26}$ The average phonon frequency $\Theta_{B}$ increases with a decrease in the number of monolayers. The broadening of the PL lines for all the samples are shown in Fig. 6. The result for $(4,4),(5,5)$, and $(20,20)$ samples have been fitted with Eq. (3). For the $(20,20)$ superlattice $\Theta_{\mathrm{LO}}$ is larger than $\Theta_{B}$, consistent with the theoretical model; $\Theta_{B}$ is an average of the acoustical and optical phonons, and hence smaller than $\Theta_{\mathrm{LO}}$. This trend is observed in bulk GaAs (Ref. 26) and $\mathrm{GaN}^{27}$ For the $(4,4)$ and $(5,5)$ superlattices, $\Theta_{\mathrm{LO}}$ being smaller than $\Theta_{B}$ may be an artifact. The fitting parameters are very sensitive in the low temperature region. For the $(4,4)$ and $(5,5)$ superlattices, at low temperatures, the type-II energies are altered due to the trapping of excitons in localized states while at high temperatures the carriers occupy higher-energy states; the large errors in $\Theta_{\mathrm{LO}}$ are due to the absence of accurate data at low temperatures. We also ob-

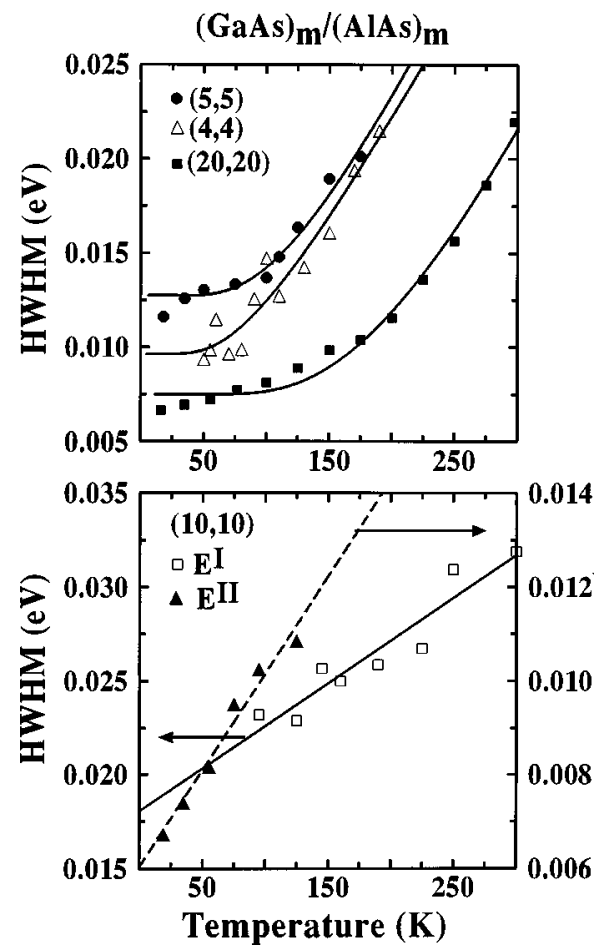

FIG. 6. Broadening of the type-I and -II transitions as a function of temperature. The solid line is a fit to Eq. (3). The broadening of the transitions in the $(10,10)$ sample has been fit with a linear equation of the form $\Gamma(T)=\Gamma_{L}+\lambda T$.

serve from the broadening parameters (Table II) that $\Gamma_{\text {ep }}$ increases with an increase in monolayer thickness. This is indicative of the change in the strength of electron-phonon interaction with change in monolayer thickness of the superlattices.

The $(10,10)$ sample is different from the other samples since in addition to the temperature shift and broadening of interband transition energy due to electron-phonon interaction, there is mixing between the $\Gamma$ and $X$ conduction bands. The broadening of the type-I and -II peaks in the $(10,10)$ have been fitted with a linear equation of the form $\Gamma(T)$ $=\Gamma_{L}+\lambda T$ because of the large scatter of the data (Fig. 6). This anomalous behavior of the broadening may be due to $\Gamma-X$ mixing since the type-I and -II transitions lie very close.

\section{TYPE-I AND -II TRANSITIONS UNDER PRESSURE}

In Fig. 7 we show typical PL spectra of $(\mathrm{GaAs})_{20} /(\mathrm{AlAs})_{20}$ measured at $16 \mathrm{~K}$ under hydrostatic pres-

TABLE II. Values of the parameters obtained by fitting the PL linewidth (half-width at half maximum) vs temperature to Eq. (3). Owing to the large scatter of data, the $(10,10)$ superlattice has been fit with a linear equation of the form $\Gamma(T)=\Gamma_{L}+\lambda T$.

\begin{tabular}{|c|c|c|c|c|c|}
\hline Sample & $\begin{array}{c}\Gamma(0) \\
\left(10^{-3} \mathrm{eV}\right)\end{array}$ & $\begin{array}{c}\text { Гep } \\
\left(10^{-3} \mathrm{eV}\right)\end{array}$ & $\begin{array}{l}\theta_{\mathrm{LO}} \\
(\mathrm{K})\end{array}$ & $\begin{array}{c}\Gamma_{L} \\
\left(10^{-3} \mathrm{eV}\right)\end{array}$ & $\begin{array}{c}\lambda \\
\left(10^{-4} \mathrm{eV} / \mathrm{K}\right)\end{array}$ \\
\hline$(4,4) E^{\mathrm{II}}$ & $9.6 \pm 0.9$ & $31 \pm 23$ & $247 \pm 106$ & & \\
\hline$(5,5) E^{\mathrm{II}}$ & $12.7 \pm 0.5$ & $54 \pm 33$ & $361 \pm 95$ & & \\
\hline$(20,20) E^{\mathrm{I}}$ & $7.5 \pm 0.3$ & $108 \pm 30$ & $647 \pm 72$ & & \\
\hline$(10,10) E^{\mathrm{II}}$ & & & & $6 \pm 0.2$ & $0.41 \pm 0.03$ \\
\hline$(10,10) E^{\mathrm{I}}$ & & & & $18 \pm 1.2$ & $0.45 \pm 0.06$ \\
\hline
\end{tabular}




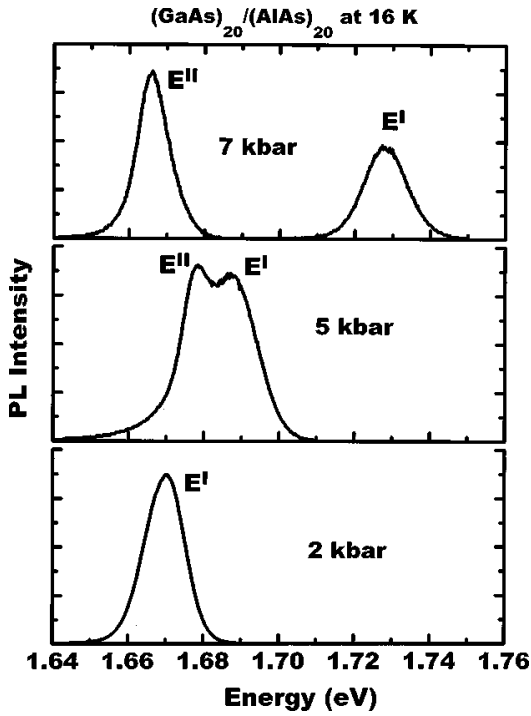

FIG. 7. PL spectra of a $(20,20)$ superlattice at three different pressures $(16 \mathrm{~K})$. The emergence of the type-II transition can be observed at the crossover pressure ( $\sim 5 \mathrm{kbar})$.

sure. The superlattice is type I at atmospheric pressure and transforms into type II at $5 \mathrm{kbar}$ as signaled by the emergence of the type-II peak. Above this pressure the superlattice is pseudodirect and the intensity of the type-I transition decreases. With an increase in pressure the high-energy peak (type-I) shifts rapidly to higher energies and the low-energy peak (type-II) moves to lower energies. Type-II emission is not observed below the crossover pressure in the PL spectra. The pressure shifts of the two transitions for the $(20,20)$ superlattice are shown in Fig. 8. The type-I and -II transitions exhibit a linear pressure coefficient of 10.1 and $-2.5 \mathrm{meV} /$ kbar, respectively, the type-I-type-II crossover occurring at $\sim 5$ kbar. The type-I and -II bands do not exhibit an anticrossing behavior due to the smallness of $\Gamma-X$ mixing $(\sim 5$ $\mathrm{meV}$ ), as expected for such a wide superlattice. For narrower superlattice, however, the effect is observable. ${ }^{28}$

Similar measurements on the $(10,10)$ superlattice displayed in Fig. 8 show that at atmospheric pressure, it is a

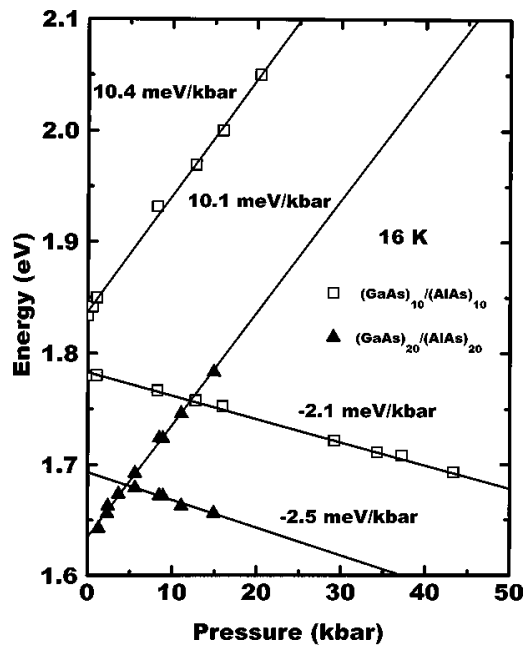

FIG. 8. Pressure dependence of the type-I and -II transitions in $(\mathrm{GaAs})_{10} /(\mathrm{AlAs})_{10}$ (squares) and $(\mathrm{GaAs})_{20} /(\mathrm{AlAs})_{20}$ (triangles) superlattices.
TABLE III. Energy positions and the pressure coefficients of the type-I and -II transitions for symmetric short-period GaAs/AlAs superlattices. The second and fourth columns show the energy positions of the type-I and -II transitions, respectively. The third and fifth columns denote the pressure coefficients for the type-I and -II transitions, respectively.

\begin{tabular}{lcccc}
\hline \hline $\begin{array}{l}\text { Monolayers } \\
(m, n)\end{array}$ & $\begin{array}{c}E^{\mathrm{I}} \\
(\mathrm{eV})\end{array}$ & $\begin{array}{c}d E^{\mathrm{I}} / d P \\
(\mathrm{meV} / \mathrm{kbar})\end{array}$ & $\begin{array}{c}E^{\mathrm{II}} \\
(\mathrm{eV})\end{array}$ & $\begin{array}{c}d E^{\mathrm{II}} / d P \\
(\mathrm{meV} / \mathrm{kbar})\end{array}$ \\
\hline$(6,6)^{\mathrm{a}}$ & 2.051 & 9.55 & 1.935 & -1.70 \\
$(10,10)$ & 1.838 & 10.4 & 1.783 & -2.01 \\
$(12,12)^{\mathrm{a}}$ & 1.807 & 10.1 & 1.761 & -2.10 \\
$(15,15)^{\mathrm{a}}$ & 1.674 & 10.5 & 1.701 & -2.04 \\
$(20,20)$ & 1.635 & 10.1 & 1.693 & -2.50 \\
Bulk GaAs $(5 \mathrm{~K})^{\mathrm{b}}$ & 1.517 & 10.7 & 2.00 & -1.34 \\
\hline \hline
\end{tabular}

${ }^{\mathrm{a}}$ From Ref. 9

${ }^{\mathrm{b}}$ From Ref. 2

type II, the $\Gamma-X$ crossover having already occurred and the energy of the pseudodirect transition, thus being lower in energy than that of the direct transition. At $P=0$, both types I and II are observed in the PL. Type-I emission shows a blueshift of $10.4 \mathrm{meV} / \mathrm{kbar}$, whereas the type-II emission is redshifted by $-2.1 \mathrm{meV} / \mathrm{kbar}$. The $(10,10)$ superlattice has higher energies for both type-I and type-II levels compared to the $(20,20)$ superlattice as a result of the increased confinement with decrease in the superlattice period. The confinement effects of the type-I transition are more pronounced than for the type-II level owing to the smaller effective mass of the conduction-band minimum.

The pressure coefficients of the energy levels in the $(10,10)$ and $(20,20)$ superlattices are consistent with the results of Holtz et al..$^{9}$ Pressure coefficients of type-I and -II recombinations are listed in Table III; $d E^{\mathrm{I}} / d P$ in GaAs/AlAs superlattices is similar to that in bulk GaAs $(10.7 \mathrm{meV} / \mathrm{kbar})$. The type-II emission has a pressure coefficient slightly different from that of $X-\Gamma$ indirect transition in GaAs $(-1.3$ $\mathrm{meV} / \mathrm{kbar}) .{ }^{10}$ Holtz et al. showed that, with decreasing thickness of the AlAs layer, the pressure coefficient of the type-II transition approaches that of the bulk GaAs value, whereas, with increasing AlAs layer thickness, $d E^{\mathrm{II}} / d P$ finally rises, reaching a constant value of $-2.0 \mathrm{meV} / \mathrm{kbar}$. They ascribe this behavior to the delocalization of the hole wave function with decreasing AlAs thickness; as a consequence, the valence band of the superlattice thus loses its square-well character, i.e., it has a single constant energy level along the growth direction. In the small barrier limit, the top of the superlattice valence band acquires the same character as in bulk tetrahedral semiconductors. Our results for the $(10,10)$ and $(20,20)$ samples are consistent with their analysis; $d E^{\mathrm{II}} / d P$ for the $(20,20)$ sample is slightly higher than that for the $(10,10)$ sample, and is close to the constant value of $-2.0 \mathrm{meV} / \mathrm{kbar}$.

\section{INTERVALLEY DEFORMATION POTENTIAL}

The type-I excitonic line in the PL spectrum of a GaAs/ AlAs superlattice is broadened by the hybridization of the $\Gamma$ exciton with the $X$ and the $L$ continua via electron-phonon coupling. Under external pressure, the $\Gamma$ conduction mini- 
mum moves up in energy and crosses the bottom of the $L$ and $X$ conduction bands of the AlAs layer. The coupling between the $\Gamma$ exciton and the $X$ and $L$ continua via intervalley scattering of the electron broadens the $\Gamma$ exciton level observed in PL. The position of the exciton relative to the continuum can be tuned with pressure. Below the crossover pressure, there are no states in the $X$ and $L$ valleys into which the $\Gamma$ electron can be scattered. Since the resonance broadening can be controlled by varying pressure, the hydrostatic pressure experiments are useful in determining the intervalley scattering rates or the intervalley deformation potential between the $\Gamma$ valley and the $X$ and $L$ valleys. Intervalley deformation potentials, which are a measure of the strength of the electron-phonon coupling, have been experimentally obtained for bulk GaAs (Ref. 29) and $\mathrm{GaAs} / \mathrm{Al}_{x} \mathrm{Ga}_{1-x} \mathrm{As}$ quantum wells ${ }^{11}$ from the broadening of the $\Gamma$ exciton under hydrostatic pressure. The intervalley deformation potential for GaAs/AlAs superlattices can be obtained from hydrostatic pressure experiments as a function of pressure, the experiments being performed at a series of fixed temperatures.

It is usually the zone-boundary phonons which cause intervalley scattering, the associated electron-phonon interactions being short range since zone-edge phonons cannot generate long-range electric fields. The matrix element for the scattering of a carrier $|\mathbf{k}\rangle$ by absorption or emission of a phonon $|\mathbf{q}\rangle$ in the absence of any external field is given by $\langle\mathbf{k} \pm \mathbf{q}|H| \mathbf{k}\rangle$, where $H$ is the electron-phonon Hamiltonian. Since $H$ has the symmetry of the phonon involved in the interaction, the transition is forbidden unless the representations belonging to the state $|\mathbf{k} \pm \mathbf{q}\rangle$ are contained in the product of the representation belonging to $|\mathbf{q}\rangle$ and $|\mathbf{k}\rangle$. In zincblende semiconductors, it can be shown ${ }^{30,31}$ that longitudinal acoustic and optical phonons leading to $\Gamma-X$ scattering contribute to the interaction, whereas transverse phonons do not participate in such an intervalley scattering. However, it has been demonstrated that the electron-phonon matrix elements show a strong dependence on the wave vector $(k)$ of the intervalley phonon, the $k$ dependence allowing the transverse acoustic TA phonons to contribute to intervalley scattering (a process not allowed at high-symmetry points). ${ }^{12}$ It is the contribution from these TA phonons which causes an apparent temperature dependence of the intervalley deformation potential. $^{12}$

The PL linewidth $\Delta$ contains information about the strength of the electron-phonon coupling, and is given by

$$
\Delta=\pi\left|M_{\Gamma X}\right|^{2} \rho_{X}(\varepsilon)+\pi\left|M_{\Gamma L}\right|^{2} \rho_{L}(\varepsilon) .
$$

Here $\left|M_{\Gamma X}\right|$ is the electron-phonon matrix element for scattering between $\Gamma$ and $X$, and $\left|M_{\Gamma L}\right|$ is that between $\Gamma$ and $L$. A detailed derivation of the above is given in Ref. 11. $\rho_{X}(\varepsilon)$ and $\rho_{L}(\varepsilon)$ in Eq. (4) are the density of states given by

$$
\rho_{X}(\varepsilon)=N_{X} \frac{m_{X}^{3 / 2} \sqrt{\varepsilon-\varepsilon_{X}}}{\sqrt{2} \pi^{2} \hbar^{3}}
$$

with a similar expression for $\rho_{L}(\varepsilon) . \varepsilon_{X}\left(\varepsilon_{L}\right)$ is the energy of the bottom of the $X(L)$ conduction band, and $N_{X}\left(N_{L}\right)$ is the number of $X(L)$ valleys; $N_{X}=3$ and $N_{L}=4$. The deformation potential $D_{\Gamma X}$ is related to the electron-phonon matrix element by

\section{$(\mathrm{GaAs})_{20} /(\mathrm{AlAs})_{20}$}

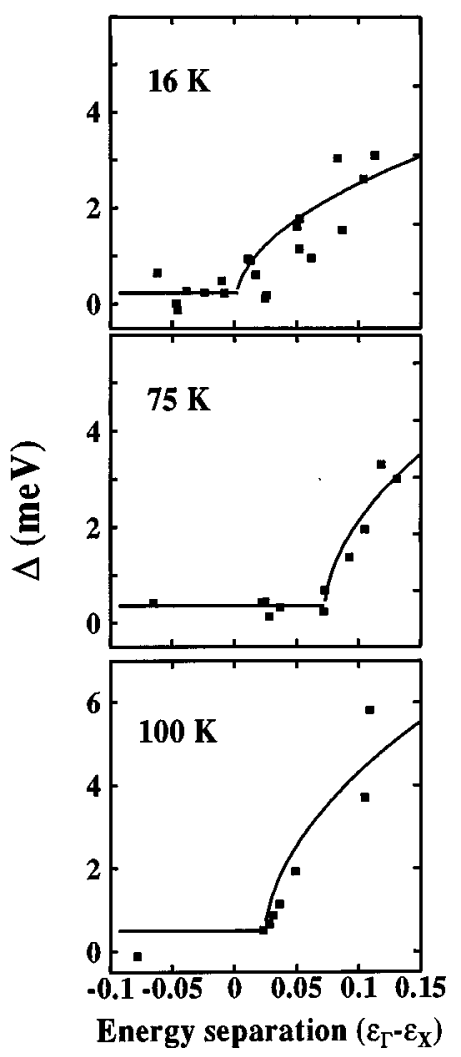

FIG. 9. Broadening of the type-I transition vs energy separation between $E^{\mathrm{II}}$ and $E^{\mathrm{I}}$ for the $(20,20)$ sample, at three different temperatures. Above crossover the linewidth is fit with a square-root function $\Delta / \sqrt{\varepsilon_{\Gamma}-\varepsilon_{X}}$.

$$
\left|M_{\Gamma X}\right|=\sqrt{\hbar /\left(2 V \rho \Omega^{x}\right)} D_{\Gamma X} .
$$

Here $V$ is the volume of the crystal, $\rho$ is the crystalline density, and $\Omega^{x}$ is the phonon frequency. From Eqs. (4), (5), and (6), the PL linewidth can be written as

$$
\Delta=\frac{\sqrt{\varepsilon-\varepsilon_{X}}}{2 \sqrt{2} \rho \pi \hbar} f(T)\left(\frac{D_{\Gamma X}^{2} N_{X} m_{X}^{3 / 2}}{\hbar \Omega^{X}}+\frac{D_{\Gamma L}^{2} N_{L} m_{L}^{3 / 2}}{\sqrt{2} \hbar \Omega^{L}}\right) .
$$

Here $\Delta$ is defined as the difference between the actual linewidth and the average linewidth below the crossover pressure $P_{C} ; f(T)=1 /\left[1-\exp \left(-\hbar \Omega / K_{B} T\right)\right]$. We assume $m_{X}$ $=0.41 m_{e}$ and $m_{L}=0.22 m_{e}$, the crystalline density $\rho$ $=5.34 \mathrm{~g} / \mathrm{cm}^{3}$ and the phonon energies as $\hbar \Omega^{X} \approx \hbar \Omega^{L}$ $\approx 30 \mathrm{meV}$, values typical for GaAs. The difference between the energy of the $\Gamma$ valley and the bottom of the $X(L)$ conduction band varies linearly with pressure; $\varepsilon-\varepsilon_{X}=\alpha_{\Gamma X}(P$ $\left.-P_{c}\right)$ and $\varepsilon-\varepsilon_{L}=\alpha_{\Gamma L}\left(P-P_{c}\right)$ where $\alpha_{\Gamma X}$ is twice as large as $\alpha_{\Gamma L} \cdot{ }^{11,32}$ This implies that $\sqrt{\varepsilon-\varepsilon_{L}}=(1 / \sqrt{2}) \sqrt{\varepsilon-\varepsilon_{X}}$ and hence the factor of $1 / \sqrt{2}$ in the second term in Eq. (7). $P_{c}$ is defined as the pressure at which the type-I and -II transitions have the same energy $\left(\varepsilon_{\Gamma}=\varepsilon_{X}\right)$; for $(\mathrm{GaAs})_{20} /(\mathrm{AlAs})_{20}, P_{c}$ $\sim 5$ kbar.

Figure 9 shows the broadening $\Delta$ for $(\mathrm{GaAs})_{20} /(\mathrm{AlAs})_{20}$ as a function $\varepsilon_{\Gamma}-\varepsilon_{X}$ at three different temperatures. The PL line shapes were fitted with Gaussians. The linewidths thus deduced increase as $\sim\left(\varepsilon-\varepsilon_{X}\right)^{1 / 2}$. The energy separation 
$\varepsilon_{\Gamma}-\varepsilon_{X}=0.15 \mathrm{eV}$ corresponds to a pressure $\sim 12 \mathrm{kbar}$. For all three temperatures, the linewidth remains almost constant below crossover pressure but changes beyond $P_{c}$. We fit the linewidth above crossover by a square-root function which changes as a function of temperature. From such fits we obtain $\Delta / \sqrt{\varepsilon-\varepsilon_{X}}$, and deduce $D_{\Gamma X}$ from Eq. (7). Experimentally, we cannot measure $D_{\Gamma X}$ and $D_{\Gamma L}$ separately; from the experimentally known value of $D_{\Gamma L}=6.5 \pm 1.5 \mathrm{eV} / \AA{ }^{33},{ }^{33}$ assuming that $D_{\Gamma L}$ remains constant with temperature, we obtain $D_{\Gamma X}=5.2 \mathrm{eV} / \AA(16 \mathrm{~K}), D_{\Gamma X}=7.3 \mathrm{eV} / \AA(75 \mathrm{~K})$, and $D_{\Gamma X}=8.2 \mathrm{eV} / \AA(100 \mathrm{~K})$. From an optical-absorption study of the exciton line broadening under pressure in GaAs, Goñi et $a .^{29}$ showed $D_{\Gamma X}$ to be temperature independent with an average value of $4.8 \mathrm{eV} / \AA$ A. Satpathy et al. ${ }^{11}$ obtained the deformation potential for GaAs as $10.7 \mathrm{eV} / \AA$ at $80 \mathrm{~K}$ from pressure-induced photoluminescence line broadening in $\mathrm{GaAs} / \mathrm{Al}_{x} \mathrm{Ga}_{1-x} \mathrm{As}$ quantum wells. We note that our values are somewhat different from those obtained in Ref. 11, since our study involved GaAs/AlAs superlattices whereas previous works were based on $\mathrm{GaAs}$ or $\mathrm{GaAs} / \mathrm{Al}_{x} \mathrm{Ga}_{1-x}$ As quantum wells.

The temperature dependence of the deformation potential is consistent with the theory of Ref. 12, where the effective deformation potential with the inclusion of TA phonons was shown to be temperature dependent; that study demonstrated that the effective deformation potentials $D_{\Gamma L}$ and $D_{\Gamma X}$ for GaAs increase up to $200 \mathrm{~K}$ and almost remain a constant above it. Their calculation yielded $D_{\Gamma X} \sim 8 \mathrm{eV} / \AA$ at $100 \mathrm{~K}$, very close to our value.

The PL line shape exhibits an asymmetry which has a temperature dependence. Asymmetry in the PL line shape is clearly observed in $(\mathrm{GaAs})_{10} /(\mathrm{AlAs})_{10}$, a type-II superlattice at atmospheric pressure; the PL spectrum recorded at $12 \mathrm{kbar}$ as a function of temperature clearly exhibits (see the insets in Fig. 10) the increasing asymmetry. The inset of Fig. 10 shows the PL spectrum for different temperatures at $12 \mathrm{kbar}$. We have fitted the spectra using the Fano line-shape analysis according to $\sim C(q+\epsilon)^{2} /\left(1+\epsilon^{2}\right)$, where $q$ is the asymmetry parameter, and $\epsilon=E-E_{0} / \Gamma ; E_{0}$ is the central peak energy and $\Gamma$ is the linewidth. The Fano line shape is due to the broadening associated with the resonance interaction of a localized level with the continuum; it is more pronounced in the $(10,10)$ superlattice as compared to that in the $(20,20)$ superlattice. We find from Fig. 10 that $q$ decreases with an increase in temperature. The asymmetry parameter $q$ is directly proportional to the average electron-phonon matrix element [Eq. (12c) of Ref. 11]. This temperature dependence of $q$ is another indication that the electron-phonon matrix element is temperature dependent, in turn leading to the temperature dependence of the intervalley deformation potential.

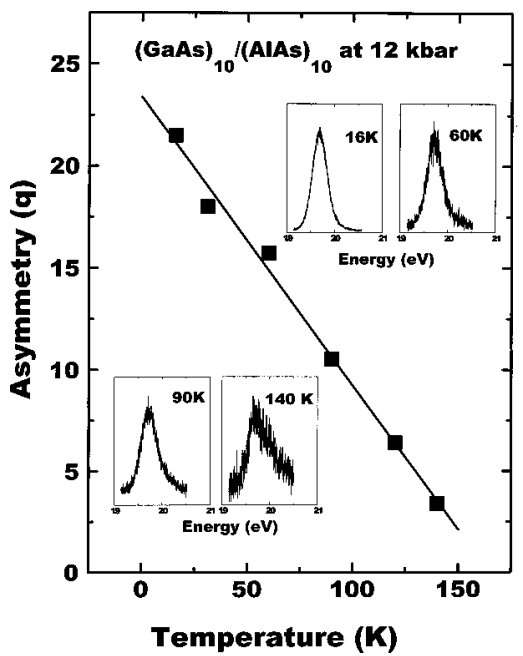

FIG. 10. Asymmetry parameter of the type-I transition as a function of temperature for the $(10,10)$ superlattice at $12 \mathrm{kbar}$. The PL line shape has been fit with a Fano line shape of the form $C(q+\epsilon)^{2} /\left(1+\epsilon^{2}\right)$. The insets show the PL spectra at various temperatures.

\section{CONCLUDING REMARKS}

The present photoluminescence spectra of a series of $(\mathrm{GaAs})_{m} /(\mathrm{AlAs})_{m}$ short-period superlattices, investigated as a function of temperature at ambient pressure as well as hydrostatic pressure accessible in a diamond anvil cell, have provided insights into (1) type-I-type-II crossover, (2) $\Gamma-X$ intervalley deformation potentials, and (3) broadening mechanisms for excitonic transitions. Experiments carried out as a function of temperature at a series of fixed hydrostatic pressures provide an opportunity to discover the temperature dependence of intervalley deformation potential. The temperature dependence of the type-I and -II transitions is described more closely by the model in which one considers Bose-Einstein statistical factors for phonon emission and absorption rather than the empirical Varshni formula.

\section{ACKNOWLEDGMENTS}

We thank S. Satpathy and S. Zollner for valuable discussions. One of us (H.R.C.) acknowledges support by the NSF under Grant No. DMR-9633107. M.C. thanks the U.S. Army for support through Grant No. DAAL03-92-0381. The work at Purdue University was supported by the National Science Foundation: Materials Research Science and Engineering Center Grant Nos. DMR 94-00415 and DMR 93-03186.

${ }^{1}$ For a review, see B. A. Wilson, IEEE J. Quantum Electron. 24, 1763 (1988).

${ }^{2}$ See, for example, S. Adachi, GaAs and Related Materials: Bulk Semiconducting and Superlattice Properties (World Scientific, Singapore, 1994).

${ }^{3}$ R. Eppenga and M. F. H. Shuurmans, Philips Tech. Rev. 44, 137 (1988); M.-P. Houng, Y. C. Chang, and W. I. Wang, J. Appl. Phys. 64, 4609 (1988).

\footnotetext{
${ }^{4}$ The effective masses (units of $m_{0}$ ) of $\Gamma$ and $X$ electrons and heavy holes are $0.0665,1.3$, and 0.34 , respectively, for GaAs; corresponding values for AlAs are 0.15, 1.1, and 0.4. The band gap (in units of $\mathrm{eV}$ ) of $\Gamma$ and $X$ conduction-band minima from the valence-band maximum are 1.511 and 1.941 for GaAs and 3.125 and 2.223 for AlAs. The band-offset ratio $\Delta E_{c}: \Delta E_{v}$ is 0.67:0.33.

${ }^{5}$ A. Jayaraman, Rev. Mod. Phys. 55, 65 (1983).
} 
${ }^{6}$ M. Chandrasekhar and H. R. Chandrasekhar, High Press. Res. 9, 57 (1992).

${ }^{7}$ M. Chandrasekhar and H. R. Chandrasekhar, Philos. Mag. B 70, 369 (1994).

${ }^{8}$ H. R. Chandrasekhar and M. Chandrasekhar, in Bandgap Tuning in Semiconductors and Heterostructures, Encyclopedia of Electronics and Electrical Engineering, edited by J. G. Webster (Wiley, New York, in press).

${ }^{9}$ M. Holtz, R. Cingolani, K. Reimann, R. Muralidharan, K. Syassen, and K. Ploog, Phys. Rev. B 41, 3641 (1990).

${ }^{10}$ M. Holtz, K. Syassen, R. Muralidharan, and K. Ploog, Phys. Rev. B 41, 7647 (1990).

${ }^{11}$ S. Satpathy, M. Chandrasekhar, H. R. Chandrasekhar, and U. Venkateswaran, Phys. Rev. B 44, 11339 (1991).

${ }^{12}$ S. Zollner, S. Gopalan, and M. Cardona, Appl. Phys. Lett. 54, 614 (1989); J. Appl. Phys. 68, 1682 (1990); Solid State Commun. 76, 877 (1990).

${ }^{13}$ A. Kangarlu, H. R. Chandrasekhar, M. Chandrasekhar, Y. M. Kapoor, F. A. Chambers, B. A. Vojak, and J. M. Meese, Phys. Rev. B 37, 1035 (1988).

${ }^{14}$ L. Viña, S. Logothetidis, and M. Cardona, Phys. Rev. B 30, 1979 (1984).

${ }^{15}$ P. B. Allen and M. Cardona, Phys. Rev. B 23, 1495 (1981).

${ }^{16}$ H. Y. Fan, Phys. Rev. 82, 900 (1951).

${ }^{17}$ E. Antončik, Czech. J. Phys. B 5, 449 (1955).

${ }^{18}$ Y. P. Varshni, Physica (Amsterdam) 34, 149 (1967).

${ }^{19}$ P. Lautenschlager, P. B. Allen, and M. Cardona, Phys. Rev. B 31, 2163 (1985); 33, 5501 (1986).
${ }^{20}$ M. Nakayama, I. Tanaka, I. Kimura, and H. Nishimura, Jpn. J. Appl. Phys. Part 1 29, 41 (1990).

${ }^{21}$ M. Kondow, S. Minagawa, Y. Inoue, T. Nishino, and Y. Hamakawa, Appl. Phys. Lett. 54, 1760 (1989).

${ }^{22}$ Y. Ishitani, S. Minagawa, and T. Tanaka, J. Appl. Phys. 75, 5326 (1994).

${ }^{23}$ Y. Ishitani, S. Minagawa, T. Kita, T. Nishino, H. Yaguchi, and Y. Shikari, J. Appl. Phys. 80, 4592 (1996).

${ }^{24}$ H. Fujimoto, C. Hamaguchi, T. Nakazawa, K. Taniguchi, K. Imanishi, H. Kato, and Y. Watanabe, Phys. Rev. B 41, 7593 (1990).

${ }^{25}$ J. Humlíček, F. Lukeš, and K. Ploog, Phys. Rev. B 42, 2932 (1990).

${ }^{26}$ P. Lautenschlager, M. Garriga, S. Logothetidis, and M. Cardona, Phys. Rev. B 35, 9174 (1987).

${ }^{27}$ C. F. Li, Y. S. Huang, L. Malikova, and F. H. Pollack, Phys. Rev. B 55, 9251 (1997).

${ }^{28}$ M.-H. Meynadier, R. E. Nahory, J. M. Worlock, M. C. Tamargo, J. L. de Miguel, and M. D. Sturge, Phys. Rev. Lett. 60, 1338 (1988).

${ }^{29}$ A. R. Goñi, A. Cantarero, K. Syassen, and M. Cardona, Phys. Rev. B 41, 10111 (1990).

${ }^{30}$ J. L. Birman, Phys. Rev. 127, 1093 (1962).

${ }^{31}$ M. L. Cohen and T. K. Bergstresser, Phys. Rev. 141, 789 (1966).

${ }^{32}$ U. Venkateswaran, M. Chandrasekhar, H. R. Chandrasekhar, B. A. Vojak, F. A. Chambers, and J. M. Messe, Phys. Rev. B 33, 8416 (1986).

${ }^{33}$ J. Shah, B. Deveaud, T. C. Damen, W. T. Tsang, A. C. Gossard, and P. Lugli, Phys. Rev. Lett. 59, 2222 (1987). 